\title{
Elliptic Curve Integral Points on $y^{2}=x^{3}+19 x-46$ \\ Jianhong Zhao ${ }^{1, \text { a }}$, Lixing Yang ${ }^{2, b}$
}

${ }^{1}$ Department of Teachers and Education, Lijiang Teachers College, Lijiang, 674199, Yunnan, China 2Department of Teachers and Education, Lijiang Teachers College, Lijiang, 674199, Yunnan, China aE-mail:312508050@qq.com, b37574990@qq.com

Keywords: Elliptic Curve; Pell equation; integer solution; common solution; Legendre symbol. Abstract. By using congruence and Legendre Symbol, it can be proved that elliptic curve $y^{2}=x^{3}+19 x-46$ has only one integer point: $(x, y)=(2,0)$.

\section{Introduction}

The positive integer points and integral points of elliptic curves are very important in the theory of number and arithmetic algebra, it has a wide range of applications in cryptography and other fields. There are some results of positive integer points of elliptic curve

$$
y^{2}=x^{3}+a x+b, a, b \in Z
$$

In 1987, D. Zagier ${ }^{[1]}$ submit the question of the integer points on elliptic curve (1) while $a=-27, b=62$, that is $y^{2}=x^{3}-27 x+62$, it counts a great deal to the study of the arithmetic properties of elliptic curves.

In 2009, Zhu H L and Chen $\mathrm{J} \mathrm{H}^{[2]}$ solved the problem what D. Zagier submitted by using algebraic number theory and $\mathrm{P}$-adic analysis method.

In 2010, By using the elementary method, $\mathrm{Wu} \mathrm{H} \mathrm{M}^{[3]}$ obtain all the integral points of elliptic curves $y^{2}=x^{3}-27 x-62$.

In 2015, Li Y Z and Cui B $\mathrm{J}^{[4]}$ solved the problem of the integer points on $y^{2}=x^{3}-21 x-90$ By using the elementary method.

In 2016 , Guo $\mathrm{J}^{[5]}$ solved the problem of the integer points on $y^{2}=x^{3}+27 x+62$ by using the elementary method.

In 2017, Guo $\mathrm{J}^{[6]}$ proved that $y^{2}=x^{3}-2 \mathbf{1} x+90$ has no integer points by using the elementary method.

Put in a nutshell, Scholars studied the integer points on elliptic curve (1) while $a_{1}=-27, b_{1}=62 ; a_{2}=-27, b_{2}=-62 ; a_{3}=-21, b_{3}=-90 ; a_{4}=27, b_{4}=62 ; a_{5}=$ $-21, b_{5}=90$.

Up to now, there is no relevant conclusions while $a=19, b=-46$.

\section{Key lemma}

Key lemma ${ }^{[7]}$ Let $D$ to be a square-free positive integer, then the equation $x^{2}-D y^{4}=1$ will have two sets of positive integer solutions $(x, y)$ at most

When $D=2^{4 s} \times 1785$, where $s \in\{0,1\}$, we can get that $\left(x_{1}, y_{1}\right)=\left(169,2^{1-s^{3}}\right)$ and $\left(x_{2}, y_{2}\right)=\left(6525617281,2^{1-s} \times 6214\right)$;

Otherwise when $D \neq 2^{4 s} \times 1785, \quad\left(x_{1}, y_{1}\right)=\left(u_{1}, \sqrt{\nu_{1}}\right)$ and $\left(x_{2}, y_{2}\right)=\left(u_{2}, \sqrt{v_{2}}\right)$, where $\left(u_{n}, v_{n}\right)$ is a positive integer solution of the Pell equation $U^{2}-D V^{2}=1$, if $x^{2}-D y^{4}=1$ has only 
one set of positive integer solution $(x, y)$ and the positive integer $n$ is suitable for $\left(x, y^{2}\right)=\left(u_{n}, v_{n}\right)$, then $n=2$ consequently; otherwise if $n$ is an even number; otherwise if $n$ is an odd number, then $n=1$ or $p$, here $p$ is a prime numbers and $p \equiv 3(\bmod 4)$.

\section{Proof of main theorem}

By using elementary method such as congruence and Legendre Symbol, the integer points on $y^{2}=x^{3}+19 x-46$ can be obtained.

\section{Theorem}

Elliptic curve

$$
y^{2}=x^{3}+19 x-46
$$

has only one integer point $(x, y)=(2,0)$.

\section{Proof of the main theorem}

Elliptic curve (2) is equivalent to

$$
y^{2}=(x-2)\left(x^{2}+2 x+23\right)
$$

\section{Primary analysis}

Obviously $(x, y)=(2,0)$ is an integer point of the elliptic curve (3), suppose $(x, y)$ is another integer point of the elliptic curve (3).

Because $x^{2}+2 x+23=x(x-2)+4(x-2)+31=(x+4)(x-2)+31$.

$\operatorname{gcd}\left(x-2, x^{2}+2 x+23\right)=\operatorname{gcd}(x-2,(x+4)(x-2)+31)=\operatorname{gcd}(x-2,31)$, and the divisor of the prime number 31 is 1 or 31 , then $g c d(x-2,31)=1$, or $g c d(x-2,31)=31$, in other words, the range of this greatest common divisor is $\{1,31\}$. as a result, we have to discuss in two cases of the elliptic curve (3):

Case I $x-2=a^{2}, x^{2}+2 x+23=b^{2}, y=a b, g c d(a, b)=1, a, b \in Z$.

Case II $x-2=31 a^{2}, x^{2}+2 x+23=31 b^{2}, y=31 a b, g c d(a, b)=1, a, b \in Z$.

\section{Discussion on Case I}

$\because a^{2} \equiv 0,1(\bmod 4)$.

$\therefore x=a^{2}+2 \equiv 2,3(\bmod 4)$.

$\therefore x^{2}+2 x+23 \equiv 2,3(\bmod 4)$.

At the same time $b^{2}=x^{2}+2 x+23 \equiv 0,1(\bmod 4)$.

Then we will get $2,3(\bmod 4) \equiv 0,1(\bmod 4)$, it is self-contradiction, this shows that (3) has no integer points.

\section{Discussion on Case II}

Divide integers into two categories as $2 \nmid a$ and $2 \mid a$ discuss separately.

First step: suppose $2 \nmid a$.

$\because 2 \nmid a \cdot \therefore a^{2} \equiv 1(\bmod 4)_{x}: x=31 a^{2}+2 \equiv 1(\bmod 4), \therefore x^{2}+2 x+23 \equiv 2(\bmod 4)$,

At the same time $b^{2} \equiv 0,1(\bmod 4)$.

$\therefore 31 b^{2}=x^{2}+2 x+23 \equiv 0,3(\bmod 4)$ it means $2(\bmod 4) \equiv 0,3(\bmod 4)$, it is self-contradiction, this shows that (3) has no integer points as well.

Second step: suppose 2 la. 
$\because 2 \mid a$, let $a=2 c, c \in Z$, and $x-2=31 a^{2} . \therefore x=124 c^{2}+2$.

Go a step further $x^{2}+2 x+23=(x+1)^{2}+22=\left(124 e^{2}+3\right)^{2}+22=31 b^{2}$, it is $\left(12 c^{2}+1\right)^{2}+352 c^{4}=b^{2}$, it is equivalent to:

$$
\left(b+12 c^{2}+1\right)\left(b-12 c^{2}-1\right)=352 c^{4}
$$

$\because 2 \mid a$, and $x-2=31 \alpha^{2}: 2\left|31 \alpha^{2}+2, \therefore 2\right| x$

$\because x^{2}+2 x+23=31 b^{2} \therefore 2+x^{2}+2 x+23 . \therefore 2+31 b^{2} . \therefore 2+b . \therefore 2 \mid b$.

Taken together, $2 \mid\left[b-\left(12 c^{2}+1\right)\right]$.

$$
\begin{aligned}
\therefore \operatorname{gcd}\left(b+12 c^{2}+1, b-12 c^{2}-1\right) & =\operatorname{gcd}\left(24 c^{2}+2, b-12 c^{2}-1\right) \\
& =2 \operatorname{gcd}\left(12 c^{2}+1, b-12 c^{2}-1\right) \\
& =2 \operatorname{gcd}\left(12 c^{2}+1, b\right) .
\end{aligned}
$$

Let $d=\operatorname{gcd}\left(12 c^{2}+1, b\right)$, Then $d|b, d| 12 c^{2}+1$, so, $d \mid\left(b+12 c^{2}+1\right)$, Therefore, $d\left[352 c^{4}\right.$, $\operatorname{gcd}\left(12 \epsilon^{2}+1,352 \epsilon^{4}\right)=\operatorname{gcd}\left(12 \epsilon^{2}+1,11\right)$

The divisor of the prime number 11 is 1 or 11 , then $\operatorname{gcd}\left(12 c^{2}+1,352 c^{4}\right)=1$, or $\operatorname{gcd}\left(12 c^{2}+1,352 c^{4}\right)=11$, in other words, the range of this greatest common divisor is $[1,11]$.

If $g c d\left(12 c^{2}+1,352 c^{4}\right)=11$.

$$
\begin{aligned}
& 12 c^{2}+1 \equiv 0(\bmod 11) \\
& \therefore 12 c^{2} \equiv-1(\bmod 11) .
\end{aligned}
$$

Because the Legendre symbol value is $\left(\frac{12 e^{2}}{11}\right)=\left(\frac{3}{11}\right)=1$, while the Legendre symbol value is $\left(\frac{-1}{11}\right)=-1$, it is self-contradiction, this shows that $g c d\left(12 c^{2}+1,352 c^{4}\right)=11$ is false. It must be $\operatorname{gcd}\left(12 c^{2}+1,352 c^{4}\right) \neq 11$.

Therefore, $\quad g c d\left(12 c^{2}+1,352 c^{4}\right)=1$.

$$
\therefore \operatorname{gcd}\left(b+12 c^{2}+1, b-12 c^{2}-1\right)=2 \text {. }
$$

Furthermore $352=2^{5} \times 11$, equation (4) can be divided into:

$$
\left\{\begin{array}{l}
b+12 c^{2}+1=2 g m^{4} \\
b-12 e^{2}-1=\frac{146}{g} n^{4} t \\
c=m n
\end{array}\right.
$$

Where $\operatorname{gcd}(m, n)=1, \operatorname{gcd}\left(g, \frac{g g}{g}\right)=1, g=1,11,2^{3}=8,2^{3} \times 11=88$.

From the first two formulas (5), we will get:

$$
12 c^{2}+1=g m^{4}-\frac{88}{g} n^{4}
$$

Making an equivalent of the modulus 4 on (6), we will get:

$$
1 \equiv g m^{4}-\frac{88}{g} n^{4}(\bmod 4)
$$

When $g=11,(7)$ is equivalent to:

$$
1 \equiv 3 m^{4}(\bmod 4)
$$

$\because m^{4} \equiv 0,1(\bmod 4) . \therefore 1 \equiv 3 m^{4}(\bmod 4) \equiv 0,3(\bmod 4)$.

It is self-contradiction, this shows that (8) is impossible.

When $g=2^{3} \times 11$, (7) is equivalent to:

$$
1 \equiv-n^{4}(\bmod 4)
$$

$\because n^{4} \equiv 0,1(\bmod 4) . \quad \therefore 1 \equiv-n^{4}(\bmod 4) \equiv 0,3(\bmod 4)$. 
It is self-contradiction, this shows that (9) is impossible.

When $g=2^{3}$, the (6) is equivalent to $12 c^{2}+1=8 m^{4}-11 n^{4}$, from $c=m n$ and $12 c^{2}+1=g m^{4}-\frac{38}{g} n^{4}$, we will get:

$$
12 m^{2} n^{2}+1=8 m^{4}-11 n^{4}
$$

(10) is equivalent to:

$$
\left(4 m^{2}-3 n^{2}\right)^{2}=31 n^{4}+2
$$

Making an equivalent of the modulus 4 on (11), we will get:

$$
\left(4 m^{2}-3 n^{2}\right)^{2} \equiv\left(n^{4}+2\right)(\bmod 10)
$$

We will get $2 \nmid n$ from (10), and $2 \nmid 4 m^{2}-3 n^{2}$ from (11).

$\therefore n^{4} \equiv 1,5(\bmod 10)$.

On the other hand, $\left(4 m^{2}-3 n^{2}\right)^{2} \equiv 1,5,9(\bmod 10)$.

$\therefore n^{4}+2 \equiv 3,7(\bmod 10)$.

Taken together, we will get:

$1,5,9 \equiv\left(4 m^{2}-3 n^{2}\right)^{2} \equiv n^{4}+2 \equiv 3,7(\bmod 10)$. It is self-contradiction, this shows that (12) is impossible.

When $g=1$, the (6) is equivalent to $12 c^{2}+1=m^{4}-88 n^{4}$, from $c=m n$ and $12 c^{2}+1=g m^{4}-\frac{38}{g} n^{4}$, we will get:

$$
12 m^{2} n^{2}+1=m^{4}-88 n^{4}
$$

(13) is equivalent to:

$$
\left(m^{2}-6 n^{2}\right)^{2}=124 n^{4}+1
$$

Let $s=m^{2}-6 n^{2}, s \in Z^{+},(14)$ is equivalent to:

$$
s^{2}=124 n^{4}+1
$$

Let $r=2 n^{2}, r \in Z^{+},(15)$ is equivalent to:

$$
s^{2}-31 r^{2}=1
$$

We know that (15) has only one positive integer point from the Key lemma, suppose $(s, n)$ is the positive integer point, then the Pell equation (16) has positive integer point $(s, r)=\left(s, 2 n^{2}\right)$.

$(1520,273)$ is a fundamental solution of the Pell equation (16), therefore all of the positive integer point can be represented as:

$$
s_{k}+r_{k} \sqrt{31}=(1520+273 \sqrt{31})^{k}, k \in Z^{+} .
$$

Therefore all of the positive integer point of (11) satisfied:

$$
\begin{gathered}
\left(m^{2}-6 n^{2}\right)+2 n^{2} \sqrt{31}=(1520+273 \sqrt{31})^{k}, k \in Z^{+} . \\
\therefore m^{2}-6 n^{2}=s_{k}, 2 n^{2}=r_{k}, k \in Z^{+} . \\
\therefore n_{k+2}=3040 r_{k+1}-r_{k}, r_{0}=0, r_{1}=273 .
\end{gathered}
$$

Making an equivalent of the modulus 2 on recurrent sequence (19), we will get the residue class sequence $0,1,0,1, \cdots$, cycle for 2 .

And just when $k \equiv 1(\bmod 2), \quad r_{k} \equiv 1(\bmod 2)$.

Just when $k \equiv 0(\bmod 2), \quad r_{k} \equiv 0(\bmod 2)$.

$\because 2 n^{2}=r_{k}, \therefore r_{k}$ is even. $\therefore k \equiv 0(\bmod 2)$.

We can get $k=2$ or $2 \nmid k$ from the Key lemma. 
So, we will get

$$
\begin{aligned}
m^{2}-6 n^{2}+n^{2} \sqrt{124} & =m^{2}-6 n^{2}+2 n^{2} \sqrt{31} \\
& =(1520+273 \sqrt{31})^{2} \\
& =4260799+829920 \sqrt{31} .
\end{aligned}
$$

Taken together, we will get:

$m^{2}-6 n^{2}=4260799,2 n^{2}=829920, n^{2}=414960$. All appearance it has no integer points, this shows that equation (15) has no integer points.

In conclusion, elliptic curve $y^{2}=x^{3}+19 x-46$ has only one integer point $(x, y)=(2,0)$.

\section{References}

[1] D. Zagier. Lager Integral Point on Elliptic Curves [J]. Math Comp, 1987,48:425-436.

[2] Zhu H L, Chen J H. Integral point on $y^{2}=x^{3}+27 x-62$ [J]. Math Study, 2009, 42(2): 117-125.

[3] Wu H M . Points on the Elliptic Curves $y^{2}=x^{2}-27 x-62$ [J] . J Acta Mathematica Curve Sinica,2010,53(1):205-208.

[4] Li Y Z, Cui B J. Points on the Elliptic $y^{2}=x^{2}-21 x-90$ [J]. Journal of Yanan University (Natural Science Edition), 2015, 34(3):14-15.

[5] Guo J. The Integral Points on the Elliptic Curve $y^{2}=x^{2}+27 x+62$ [J]. Journal of Chongqing Normal University (Natural Science), 2016,33(5): 50- 53.

[6] Guo J. The Positive Integral Points on the Elliptic Curve $y^{2}=x^{3}-21 x+90$ [J]. Mathematics in Practice and Theory,2017,47(8):288-291.

[7] Togbé A.,Voutier P.M., and Walsh P.G.,Solving a family of Thue equations with an application to the equation $x^{2}-d y^{2}=1$.Acta. Arith.,2005,120(1) :39-58. 